\title{
ASTROMETRY IN THE FUTURE
}

\author{
P. K. SEIDELMANN \\ U.S. Naval Observatory \\ Washington, DC, USA
}

\begin{abstract}
The introduction of the extragalactic reference frame and the availability of the Hipparcos and Tycho catalogs will have major impacts on changing the accuracy levels of astrometry in the future.

There is a need for accurate densification of the reference frames down to at least $20^{\text {th }}$ magnitude. Also accurate space motions and distances will be required. The ground based observations must be supplemented by space missions. In addition to traditional positional astrometry, the scientific applications of planetary detection, distance scales, multiple star systems, mass detections, solar system motions and geodesy will be advanced by the new accuracies.
\end{abstract}

\section{Introduction}

This is a time of change in astrometry. The Hipparcos and Tycho catalogs are almost available and the extragalactic reference frame is being obtained. In both cases milliarcsecond accuracies are being introduced and some previous astrometric activities are becoming obsolete; however, the future needs and applications of astrometry are significant. Astrometric programs, plans, methods, observational techniques, and data handling methods must change to be part of the astrometry of the future.

Specifically, the changes in astrometry currently taking place include the replacement of the primary reference frame, defined by bright stars on the dynamical reference frame, by the extragalactic reference frame based on distant radio sources. Charge coupled devices (CCDs) have replaced photographic plates as the detectors of choice. Optical interferometers are replacing transit circles. Space-based catalogs from Hipparcos are replacing ground based star catalogs. The Internet and CD ROMs have replaced magnetic tapes and floppy disks as mediums of data transfer. The wavelengths are no longer restricted to the optical, but now include radio, infrared, millimeter and ultraviolet. 


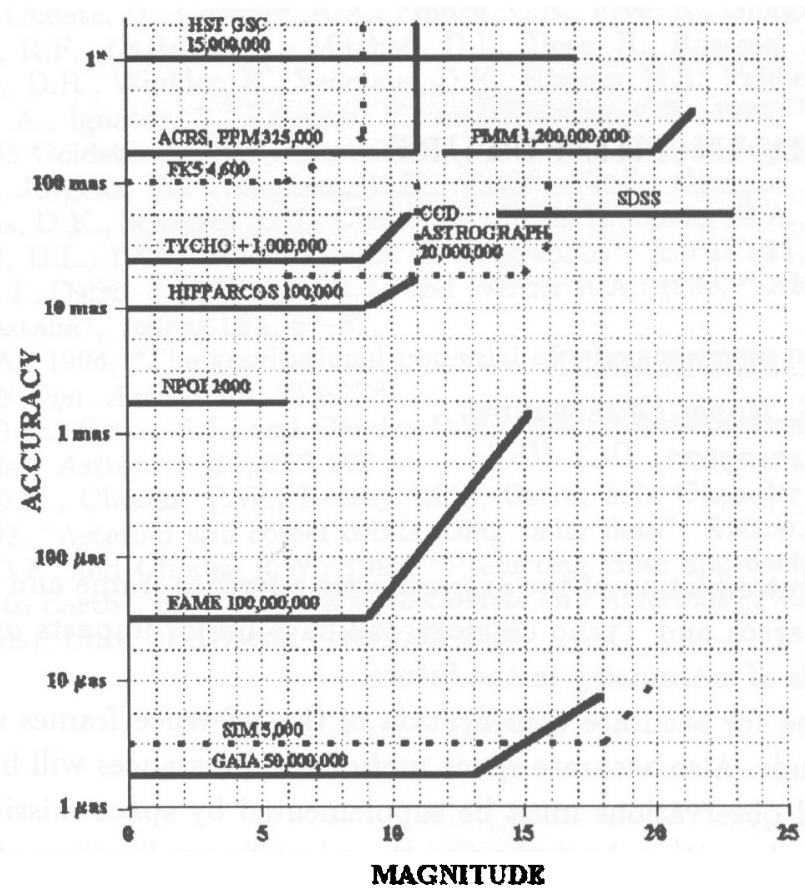

Figure 1. The historical and future development of star catalogs is plotted in terms of accuracy versus year. The number of stars in each catalog is indicated. This figure is an adaption from Hog (1995).

\section{Impact of Change}

In the past the fundamental reference frame was based on transit circle observations of bright stars, which were oriented in the dynamical reference frame of the solar system. The FK5 star positions were accurate to about 50 milliarcseconds (mas) with proper motions accurate to about 2 mas/year. Photographic plates were used to extend the reference frame down to $11^{\text {th }}$ magnitude. The ACRS and PPM catalogs combined all of these data to provide an accuracy of about 100 mas with proper motions of 3 mas/year accuracy. The accuracies given above were at the epoch of the observational data, which was about 40 years ago, so the accuracies at the current time are significantly poorer.

Very Long Baseline Interferometry (VLBI) observations of radio sources have provided milliarcsecond positional observations of the sources and, as a result, accurate Earth orientation parameters and space-fixed extragalactic reference frame. The FK5 (optical) reference frame is based on bright stars with proper motions, defined in terms of the dynamical reference frame with a moving equinox, and determined to an accuracy of about 100 mas. It is being replaced by an extragalactic reference frame based on distant 


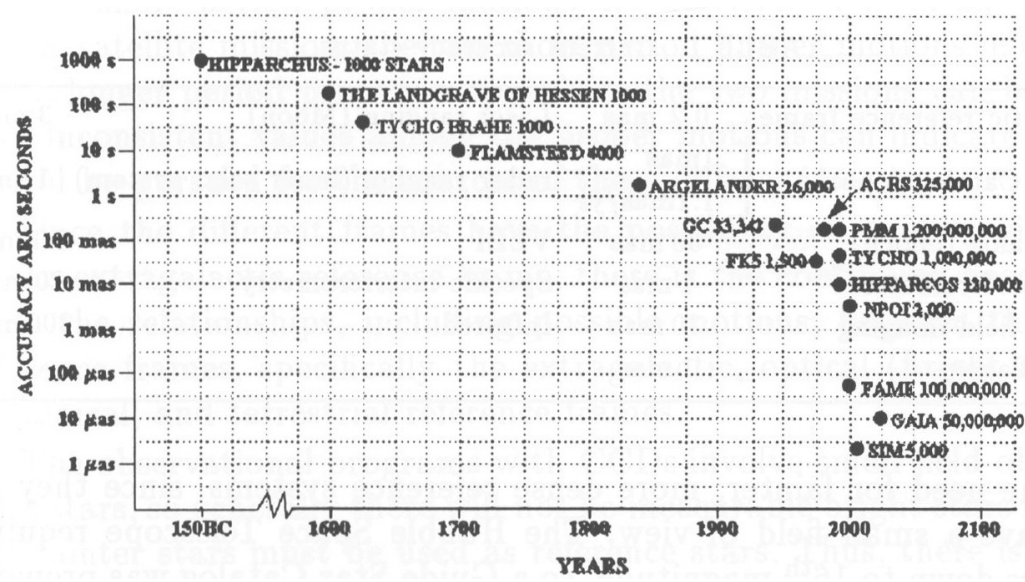

Figure 2. The present near future, and projected astrometric catalogs are plotted in accuracy versus star magnitude.

radio sources with possible changes in structure, defined as space fixed by 400-600 fundamental sources, with axes to an accuracy of about 0.2 mas. For the fundamental reference frame the dynamical system defined by solar system observations, which determine the equatorial and ecliptic planes, is replaced by a kinematic system defined by Earth-based observations of extragalactic sources which determine the equatorial pole and its motions. The dynamical system is still required for solar system dynamics.

The Hipparcos Star Catalog will provide over 100000 stars at the 1 mas level at the epoch of observations (1992) with approximately 1.1 mas/year accuracy in the proper motions for stars brighter than $9^{\text {th }}$ magnitude. So, the accuracy will be degraded to approximately 7 mas when it becomes available in 1997. It will continue to degrade unless observations are made to maintain the accuracy of the optical reference frame. Ground based optical interferometers should be able to make astrometric observations of 1 mas accuracy and maintain the optical reference frame. In addition, the Hipparcos project will provide the Tycho catalog which has approximately one million stars at 30 mas accuracy, but without proper motions. All these stars are brighter than $11^{\text {th }}$ magnitude. The historical and future accuracies of star catalogs are shown in Figure 1, adapted from Hog (1995). The present and near future astrometric catalogs in terms of accuracies and magnitude ranges are illustrated in Figure 2. The accuracies currently being achieved are given in Table 1 . As a result, observations of brighter stars to tenths of arcseconds, such as those made with visual transit circles, will no longer be useful.

The quantum efficiencies of charge coupled devices (CCDs) provide more accurate positional observations of fainter sources, but CCDs also 
TABLE 1. Current accuracy status.

\begin{tabular}{|c|c|c|c|}
\hline Extragalactic reference frame & $0.2 \mathrm{mas}$ & Laser ranging (Moon) & $3 \mathrm{~cm}$ \\
\hline Hipparcos Catalog (epoch) & $\begin{array}{l}1 \mathrm{mas} \\
1.1 \mathrm{mas} / \mathrm{yr}\end{array}$ & radar ranging (inner solar system) & $1 \mathrm{~km}$ \\
\hline Tycho Catalog (epoch) & 30 mas & VLBI & $0.1 \mathrm{mas}$ \\
\hline Optical interferometer & $1 \mathrm{mas}$ & Speckle interferometry & $20 \mathrm{mas}$ \\
\hline $\begin{array}{l}\text { CCD wide field imaging } \\
\text { CCD parallaxes }\end{array}$ & $\begin{array}{l}30 \mathrm{mas} \\
1 \mathrm{mas}\end{array}$ & Infrared & $200 \mathrm{mas}$ \\
\hline
\end{tabular}

impose the need for fainter, more dense reference systems, since they generally have a small field of view. The Hubble Space Telescope required guide stars down to $16^{\text {th }}$ magnitude, so a Guide Star Catalog was prepared from Schmidt plates covering the entire sky. Due to the distortions at the edges of the plates the accuracies of this catalog varied from 0.5 to $2^{\prime \prime}$ or worse. The catalog also did not have proper motions, since it was based on a single epoch of plates.

In addition to VLBI observations at radio wavelengths and CCD observations at optical wavelengths, infrared detector arrays are now becoming available and there are plans for millimeter wavelength antenna arrays. So astrometry has expanded across the frequency spectrum.

\section{Astrometry Needs}

Accepting the changes and their impact on astrometry, the new needs for astrometry must also be recognized. The radio reference frame must be maintained; thus, positional observations must continue and observations to detect possible changes in source structure must be made periodically. Positional changes, presumably due to structural changes, have been detected at the 25 microarcseconds per year level. The optical reference frame, which will continue to be widely used for optical observations, navigation and guidance will require subsequent observations at the accuracy level of Hipparcos, or better. The logical means of achieving this is by ground-based optical interferometry, CCD survey programs, or another astrometric satellite, such as the proposed FAME, LIGHT, or DIVA. (Seidelmann et al., 1995; Yoshizawa et al., 1997; Bastian et al. 1997)

One aspect of the maintenance of the optical reference frame should receive special attention, namely the proper motions. Historically, proper motions have been determined from observations made at widely separated epochs, 40-100 years apart. Hipparcos has determined proper motions over a short (2 year) time period. If the star is double, or has an unseen companion, the short and long period proper motions can differ significantly. 
Also, if short period proper motions are determined from another astrometric satellite mission, the two short period proper motions may disagree and a longer period proper motion from the two missions can be determined. Inconsistent values among the proper motions can indicate stars that should be pursued for the sources of these differences.

Since the different frames have the possibility of motion with respect to the extragalactic reference frame, there is the continuing need to determine the relationships, including possible motions, between the different reference frames, specifically the extragalactic, optical (bright and faint), dynamical, and terrestrial reference frames.

The observational programs with CCDs involve small field observing of faint stars, so generally there will not be measurable bright stars in the field and fainter stars must be used as reference stars. Thus, there is an urgent need to accurately densify the optical reference frame to fainter stars. A CCD survey of the sky to 15 or $16^{\text {th }}$ magnitude could densify the Hipparcos catalog at about the 30 mas accuracy level. (CCD Astrograph on Figure 2). The USNO is currently measuring the available faint survey plates, Palomar Sky Surveys I and II, and Southern Hemisphere UK and ESO plates, to provide a massive all-sky catalog with proper motions to $21^{\text {st }}$ magnitude at about the 200 mas accuracy level (PMM on Figure 2). This will require the densified intermediate optical catalog to accurately tie the faint catalog to the extragalactic reference frame.

Astrometric catalogs have traditionally included only positions and proper motions. It is necessary to now consider space positions and motions, Thus, parallaxes, or distances, and radial velocities are also needed for the star positions. It should be recognized that the distance scale is a primary astrophysical interest and a major justification for astrometric observational programs.

With improved accuracies more stars are detected as double, or in multiple star systems, and there is a greater need for accurate observations and orbital data. Speckle interferometry and optical interferometers provide relative observations at the 20 mas level.

In addition to space coordinates and motions, accurate astrometry requires photometry so that corrections for refraction and detector sensitivities can be properly incorporated. For guidance and detection programs, there is a need to extend beyond the visual wavelengths to the infrared and the ultraviolet. There are a number of programs under consideration for improving the accuracies, or the quantity, of stars in infrared catalogs. Essentially, the sky looks very different in the infrared than the optical wavelengths. Faint objects in one can be bright in the other. A currently available astrometric infrared star catalog is the Catalog of Positions of Infrared Stellar Sources (CPIRSS) (Hindsley and Harrington, 1994). This has 
been prepared by determining the identifications between the IRAS sources and the ACRS catalog, matching stellar spectra structure to ensure identification, and determining the relative magnitudes at different flux levels. Observations are necessary to validate this approach and to detect the exceptions to the magnitude predictions.

Having discussed stellar astrometry, the observations of moving objects need consideration. To maintain the accuracy of the solar system ephemerides, continuing accurate observations of solar system objects are needed. Among the planets, observations of Mars, Neptune, and Pluto are the most urgently needed; Mars due to the perturbations by the minor planets, Neptune due to lack of closure of its orbit to date, and Pluto due to the very short arc of observations and the relative inaccuracy of the early observations. Where possible, radar observations can provide kilometer accuracy range measurements; laser ranging to the Moon can achieve three centimeter accuracies. Observations of lesser accuracy do not contribute anything.

Continuing observations of planetary satellites are required with emphasis on those where accuracy is required for space missions and those whose motion is not well known or where there is interaction with others, or with rings. Satellite observations can provide the most accurate positional observations for the outer planets. The minor planets require ephemeris maintenance observations as needed and accurate observations for space missions and mass determinations. A primary source of uncertainty in solar system ephemerides and the reason for continuing emphasis on Mars observations is the perturbations by the minor planets, whose masses are not accurately known. Five minor planets have determined masses, but some may only be accurate at the $50 \%$ level. The use of uncertain densities, sizes, and shapes to approximate the masses of minor planets can not provide accurate perturbation determinations. Thus, there is a need for a systematic approach to determine accurate masses for different categories of minor planets and for improved knowledge of the shapes and sizes of the larger minor planets.

There is a special category of objects of particular interest, namely Near Earth Orbit (NEO) objects. These comets and minor planets could collide with the Earth as Comet P/Shoemaker Levy IX impacted Jupiter. The NEO objects present some special requirements. One is the detection of faint objects which may have small, or large, angular motion. For this a faint comprehensive star catalog is needed both for reference stars and to help detect moving objects. Then rapid follow up observations are needed to determine an initial orbit. Finally, accurate astrometric observations are needed for the determination of a very accurate orbit to predict exactly how close the object may come to the Earth in the future.

There is also the need for accurate angular observations of artificial satellites. The continuously increasing numbers of objects in Earth orbit 
require improved accuracies for the observations of these objects and improved predictions of their future motions. This is true for collision avoidance and for better use of available space for certain types of satellites, like geosynchronous communication satellites.

The availability of astrometric catalogs needs to be considered. How can all the astrometric data be organized and made available to everyone who needs it? There are massive new data sets which will be available soon, such as the faint star data from the Precise Measuring Microdensitometer (PMM), the 2 MASS infrared data, and the Sloan Digital Sky Survey observations. We need to think in terms of a massive database of comprehensive information that could be available to everyone. This database should include positions, motions, photometry, duplicity, variability, and type. It should contain the best values on a single reference frame. For some people this may be viewed as a massive catalog of faint stars. For others this will be a means for analyzing massive new sets of data. The database needs to be organized such that users can efficiently extract subsets of data. Whether the data should be stored centrally and available by worldwide web or widely distributed is an operational consideration.

The IAU Working Group on Astronomical Standards has been considering the availability of standardized software. The increased accuracies require equivalent levels of accuracy in the methods and algorithms underlying the software. I think this is a logical step for standardization and economy. However, this is going to require means of validating, checking and testing the software and the education of the users. The blind use of a black box software package is an invitation to disaster. The standardized software must be consistent with a standardized system of constants and definitions based on the theory of relativity. Changes must be introduced in a rational and timely manner.

\section{Astrometry Applications}

It is advisable to keep in mind the applications for astrometry, or what are the organizational and scientific benefits from astrometry. The original purpose still exists, to provide navigation and guidance by means of stellar positions, now more for spacecraft than for ships. Likewise, reference frames are still needed as discussed previously. There is also the need for accurate relationships among the reference frames in different wavelengths. Related to the reference frame are the Earth orientation parameters which are necessary for many purposes.

The calibration of the distance scale by means of accurate parallax measurements of Cepheids, R.R. Lyrae stars, O, B, and A stars, and population II subdwarfs would be a significant contribution to astrophysics from astrometry. Similarly, an accurate determination of the parallaxes and proper 
motions of stars in the vicinity of the Sun permits the determination of the thickness and density of the galactic disk. This can contribute definitive information concerning the missing mass question.

There are apparently a number of extrasolar planetary systems of various descriptions. With sufficient accuracy and time astrometric detection of some systems is possible. A number of space missions for this purpose have been proposed. Mr. Dan Golden, the Administrator of NASA, considers planetary detection one of NASA's primary goals.

Near Earth Objects could be considered a part of the space surveillance efforts, although the accuracies and rates of motion are different. With the increased accuracies and smaller sizes and magnitudes of objects to be observed, astrometry becomes more critical for space surveillance activities. CCDs provide a means of combining the tracking of the telescope with the read out of the CCD to obtain images of stars and satellites to observe the relative position of the satellite. Space Exploration requires accurate astrometry for mission planning, navigation and guidance to the targets, and accurate knowledge of the positions and motions of the targets.

\section{Observational Techniques}

With only a few exceptions, such as large fields of view surveys and bright objects, the CCD has replaced the photographic plate as the astrometric detector of choice. The advantages of the CCD include increased efficiency, wide dynamic range, immediate digitization, no adverse environmental impact, improved accuracy, scanning capability, and digital processing of the images.

Optical interferometry promises to offer the most accurate means (1 mas) for positioning and imaging observations of stars brighter than $10 \mathrm{mag}$ from the ground. However, these interferometers are expensive and complex, if the full accuracy is to be achieved. Astrometric observations require a metrology system in order to know the ground baselines precisely.

Very Long Baseline Interferometry (VLBI) is the proven technique for radio astrometry, although it is very expensive to operate. In addition to observations of extragalactic sources, VLBI has demonstrated its capability to observe geosynchronous artificial satellites at the $3 \mathrm{~m}$ accuracy level.

Space astrometry has been demonstrated by Hipparcos and the Hubble Space Telescope. There are many proposed space astrometry missions, but these are very expensive. The optical missions can be divided into the scanning type that can observe many stars covering the entire sky accurately and the pointed type that can observe a limited number of selected stars more accurately. Table 2 lists the many space missions that have been proposed. The SIM and GAIA projects are in study phase with some prospects for realization. Since SIM is a pointed mission for a limited number 
of stars, a scanning astrometric instrument between Hipparcos and GAIA with accuracies of about 50 microarcseconds would provide excellent scientific benefits. The most likely means of accomplishing an optical astrometry mission in the next decade is through an international collaborative effort.

The VSOP and Radioastron missions are scheduled to launch a radio antenna into space shortly to provide Earth to space VLBI capabilities, thus providing a much longer baseline for astrometry.

The millisecond pulsars are a new data type that offer some potential new capabilities. The reduction of these observations is very sensitive to the accuracy with which the motion of the solar system barycenter is known. Thus, they become an accuracy test of the ephemerides and, with sufficient observations over time, they might be used as a means of improving the ephemerides. For the reduction of the observations the pulsar positions must be known with respect to the dynamical reference frame. Some millisecond pulsars can be observed by VLBI, so their positions can be determined with respect to extragalactic reference frame, thus providing an accurate tie between the dynamical and extragalactic reference frames. Optical observations from the ground and with Hubble Space Telescope of the millisecond pulsars can provide a tie to the optical reference frame. In addition, if enough millisecond pulsars are observed over a period of time, they have the potential of providing an accurate independent time source.

Speckle interferometry has proven itself as a very accurate method of measuring double star separations and positions. Recording images 30 times per second freezes the atmospheric effects and gives many measurements. This, along with long baseline optical interferometry, will provide a means for investigating the many double stars that are anticipated to be detected in the near future.

The infrared detectors, such as Insb, are becoming available and offer the capability of observing the sky at various infrared wavelengths. There are a number of observational surveys planned such as 2MASS and DENIS, which should provide initial infrared observational catalogs.

The Global Positioning System (GPS) can be used for observations of the motions of positions on the surface of the Earth. Observations can be obtained for many locations, very frequently and for little cost. Thus, GPS provides the majority of the solution for polar motion and it is being investigated for UT1 determination. This could reduce the amount and frequency of VLBI observations for Earth Orientation. In addition, GPS observations are very useful for geodetic studies and for spacecraft tracking.

\section{Conclusions}

Astrometry is in a time of change. The extragalactic reference frame is replacing the Fundamental Catalog reference frame and the dynamical re- 
ference frame. The accuracy levels have changed from 100 to 0.1 milliarcseconds. CCDs have replaced photographic astrometry.

Hipparcos and Hubble Space Telescope have demonstrated the capabilities of space astrometry and the Hipparcos catalog, after correct alignment, will be the optical reference catalog.

However, Hipparcos has not completed all astrometric activities, rather it has provided the stimulus for new astrometric activities.

\section{References}

Bastian, U., Hog, E., Mandel, H., Quirrenback, A., Roser, S., Schalinski, C., Schilback, E., Seifert, W., Wanger, S., and Wicenec, A.: 1997, "DIVA, an interferometric minisatellite for astrometry and photometry", Astron. Nachr., in preparation.

Chubey, M.S., Paskkov, V.S., Kopylov, I.M., Kirian, T.R., Nickiforov, V.V., Markelov, S.V, and Ryadchenko, V.P.: 1995, "On the registration system of the AIST-project", Astronomical and Astrophysical Objectives of Sub-Milliarcsecond Optical Astrometry (E. Hog, P.K. Seidelmann, eds), Kluwer, Dordrecht, 323-326.

Eichhorn, H.: 1974, Astronomy of Star Positions, Ungar, New York.

Hindsley, R.B. and Harrington, R.S.: 1994, "The U.S. Naval Observatory Catalog of positions of infrared stellar sources", Astron J. 107, 280-6.

Hog, E.: 1995, "A new era of global astrometry II. A 10 microarcsecond mission", Astronomical and Astrophysical Objectives of Sub-Milliarcsecond Optical Astrometry (E. Hog, P.K. Seidelmann, eds), Kluwer, Dordrecht, 317-322.

Johnston, K.J., Seidelmann, P.K., Reasenberg, R.D., Babcock, and R., Phillips, J.D.: 1995, "Newcomb astrometric satellite", Astronomical and Astrophysical Objectives of Sub-Milliarsecond Optical Astrometry (E. Hog, P.K. Seidelmann, eds), Kluwer, Dordrecht, 331-334.

Kopylov, I.M., Gorshanov, D.L., and Chubey, M.S.: 1995, "Photometry facilities of the AIST space project", Astronomical and Astrophysical Objectives of SubMilliarcsecond Optical Astrometry (E. Hog, P.K. Seidelmann, eds), Kluwer, Dordrecht, 327-330.

Lindegren, L. and Perryman, M.A.C.: 1995, "A small interferometer in space for global astrometry: The GAIA concept", Astronomical and Astrophysical Objectives of Sub-Milliarcsecond Optical Astrometry (E. Hog, P.K. Seidelmann, eds), Kluwer, Dordrecht, 337-344.

Seidelmann, P.K., Johnston, K.J., Urban, S., Germain, M., Corbin, T., Shao, M., Yu, J., Fanson, J., Rickard, L.J., Weiler, K., and Davinic, N.: 1995, "A Fizeau optical interferometer astrometric satellite", in: Future Possibilities for Astrometry in Space, a Joint RGO-ESA Workshop, Cambridge UK 19-21 June 1995 ESA SP-379.

Volonte, S.: 1995, "Astronomy from a Lunar base", Astronomical and Astrophysical Objectives of Sub-Milliarcsecond Optical Astrometry (E. Hog, P.K. Seidelmann, eds), Kluwer, Dordrecht, 347-350.

Yoshizawa, M., Sato, K., Nishikawa, J., and Fukushima, T.: 1997, "Two astrometric projects: MIRA (Mitaka optical and infrared interferometer array) and LIGHT (Light interferometer satellite for the studies of galactic halo tracers)", this volume.

Yershov, V.N., Chubey, M.S., Il'in, A.E., Kopylov, I.M., Gorshavov, D.L., Kanayev, I.I., and Kirian, T.R.: 1995, "Struve space astrometric system. Scientific grounds of the project" (in Russian).

Future Possibilities for Astrometry in Space, A Joint RGO-ESA Workshop, Cambridge UK 19-21 June 1995 ESA SP-379.

Frontiers of VLBI 1992, Proceedings of the International VSOP Symposium and of the mm-VLBI Workshop (H. Hirabayashi, M. Inoue, H. Kobayashi, eds), Universal Academy Press Inc., Hongo, Tokyo, Japan. 